\title{
Effects of chameleon dispense-to-plunge speed on particle concentration, complex formation, and final resolution: A case study using a bacterial ribonucleotide reductase
}

\author{
T Levitz ${ }^{1}, C$ Drennan ${ }^{1}, \mathrm{P}$ Thaw $^{2}$ \\ ${ }^{1} M I T,{ }^{2}$ SPT Labtech, Royston, Herts \\ tlevitz@mit.edu
}

The chameleon (SPT Labtech; based on the NYSBC SpotItOn) utilizes a picoliter spraying method to dispense a thin stripe of sample onto self-wicking cryo-electron microscopy (cryo-EM) grids. This method allows for extremely fast (as fast as $54 \mathrm{~ms}$ ) dispense-to-plunge (D2P) times. The fast D2P timing, combined with the unique physics of spraying and wicking, has opened a new pathway for difficult samples that display preferred orientation or complex denaturation at the air/water interface. Here, we present a case study utilizing the chameleon to ameliorate the effects of complex denaturation through faster D2P times. Using a 528-kDa bacterial ribonucleotide reductase complex, we demonstrate improved complex stability and final resolution using the chameleon at four different D2P times $(619-54 \mathrm{~ms})$ as well as trends in protein concentration for varying D2P speeds.

Acta Cryst. (2020). A76, a215 